\title{
Comunicación
}

\section{Reporte de caso: blastomicosis esplénica canina}

\author{
Case report: canine splenic blastomycosis
}

Andrés Felipe Palacios ${ }^{1,3}$, Ricardo Piñeros ${ }^{1,2}$, Paola Barato², Frank Suárez ${ }^{1,}$

\section{Resumen}

El objetivo de este artículo fue reportar y describir seis casos de blastomicosis esplénica canina entre 2013 y 2017 en dos laboratorios de patología en Bogotá, Colombia (Laboratorio de Anatomopatología de la Universidad de La Salle-Bogotá, laboratorio de patología de la empresa CORPAVETC). Las muestras fueron obtenidas por esplenectomía consecuente a sospecha de enfermedad neoplásica. Los diagnósticos patológicos fueron considerados como hallazgos accidentales de esplenitis micótica causada por esporas de Blastomyces, pues no hubo evidencia clínica o laboratorial de enfermedad sistémica conjunta.

Palabras clave: blastomicosis; esplenitis; esplenomegalia; micosis esplénica

\section{Abstract}

The aim of this report was to describe six cases of canine splenic blastomycosis between 2013 and 2017 in two pathology laboratories in Bogotá, Colombia (Anatomopathology Laboratory of the University of La Salle-Bogotá, pathology laboratory of CORPAVET(C). The samples were obtained by splenectomy consistent with the suspicion of neoplastic disease. The pathological diagnoses were considered as accidental findings of fungal splenitis caused by Blastomyces spores, as there was no clinical or laboratory evidence of joint systemic disease.

Key words: blastomycosis; splenitis; splenomegaly; splenic mycosis

${ }^{1}$ Laboratorio de Anatomopatología, Facultad de Ciencias Agropecuarias, Programa de Medicina Veterinaria, Universidad de La Salle, Bogotá, Colombia

_Corporación Patológica Veterinaria- CORPAVET, Bogotá, Colombia

${ }^{3}$ E-mail: apalacios29@unisalle.edu.co

Recibido: 31 de julio de 2018

Aceptado para publicación: 27 de febrero de 2019 


\section{INTRODUCCIÓN}

La blastomicosis canina está clasificada entre las micosis sistémicas, de presentación granulomatosa y supurativa crónica, causada por el hongo dimórfico Blastomyces dermatitidis. El órgano más afectado es el pulmón por ser un patógeno de ingreso aerógeno; sin embargo, suele invadir otros órganos, generando lesiones tisulares características de la enfermedad (Quinn et al., 2003; McGavin y Zachary, 2007; Sánchez y Cabanilla, 2010). La enfermedad afecta diversas especies de mamíferos (Raymond et al., 1997; AEMV, 2014), habiendo sido descrita en el humano desde finales del siglo XIX (Castellani, 1928; Acha y Szyfres, 2001).

El diagnóstico de la blastomicosis por excelencia es la identificación de Blastomyces mediante cultivo del hongo, citología o histopatología (Stenske, 2008; Ramanan et al., 2017) y tinciones especiales cómo la tinción de Grocott (GMS), la tinción de PAS (periodic acid-schiff) y la tinción de hematoxilina y eosina (H-E) (Brömel y Sykes, 2005; Guarner y Brandt, 2011; Latimer et al., 2005; Patel et al., 2010; Ramanan et al., 2017). El agente suele encontrarse asociado a una reacción granulomatosa o piogranulomatosa, que junto a la presencia de esporas de Blastomyces permite realizar el diagnóstico (Guarner y Brandt, 2011; Couto y Nelson, 2014). Por otro lado, se dispone de otras pruebas serológicas como inmunodifusión, radioinmunodifusión y prueba de ELISA; sin embargo, la sensibilidad es baja y la especificidad no es satisfactoria (Guarner y Brandt, 2011; Ramanan et al., 2017).

Clínicamente, la blastomicosis se clasifica cómo una infección micótica polisistémica que ingresa por vía aerógena a los pulmones, donde las conidias son fagocitadas por los macrófagos alveolares y transformadas a su forma esporulada, de ahí se propaga vía hematógena a diversos tejidos, entre ellos el tejido linfoide. Por ser una enfermedad sistémica, se reportan numerosos signos clínicos, siendo los más comunes la anorexia, tos, disnea, intolerancia al ejercicio, pérdida de peso, enfermedad ocular, enfermedad cutánea, depresión, cojeras y síncope, así como linfadenopatía local o generalizada y esplenomegalia (Couto y Nelson, 2014; Ettinger y Feldman, 2010). Para la orientación clínica de los casos se utiliza la radiografía torácica o ecografía (Ettinger y Feldman, 2010; Couto y Nelson, 2014; Ramanan et al., 2017)

Los médicos veterinarios se encuentran expuestos a la enfermedad mediante la transmisión por accidentes durante procedimientos de necropsia, punción con agujas contaminadas e incluso por mordedura. Así mismo, no se recomienda realizar los cultivos del hongo en laboratorios de nivel de bioseguridad menor a 3 (Acha y Szyfres, 2001; Brömel y Sykes, 2005; Ettinger y Feldman, 2010), aunque Stenske (2008) menciona que puede ser manipulado con precaución en laboratorios de bioseguridad nivel 2 .

El presente reporte no solo describe las lesiones esplénicas en casos donde el examen clínico no indica enfermedad micótica sistémica, sino en comunicar a médicos veterinarios la relevancia clínica de la enfermedad y el potencial zoonótico del agente.

\section{Materiales y Métodos}

Se recopiló la casuística histopatológica en perros con esplenitis por Blastomyces entre 2013 y 2017 de la empresa Corporación Patología Veterinaria-Corpavet $\$ y el laboratorio de Patología de la Universidad de La Salle, Bogotá (Colombia). Posteriormente se confirmó que los pacientes referidos tuvieran lesiones únicamente en el bazo. Las lesiones histopatológicas fueron fotografiadas y descritas. 


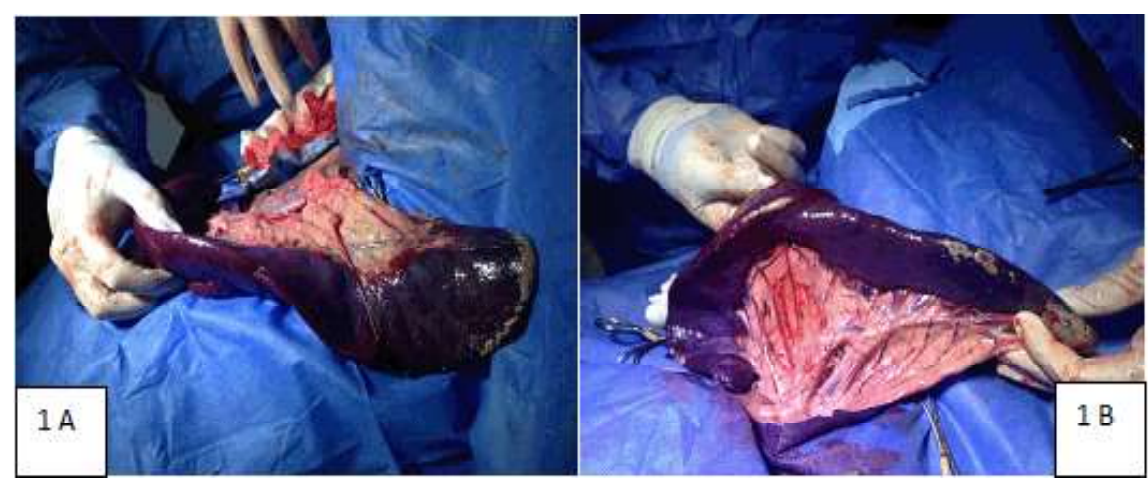

Figura 1. A-B: Procedimiento de esplenectomía, canino, hembra, Bobtail, 11 años. Se evidencia esplenomegalia, áreas irregulares, difusas y coalescentes en el extremo craneal del bazo y en el borde libre de la cápsula esplénica de color amarillento claro que sobresalen en relieve irregular

\section{Resultados y Discusión}

Se encontraron seis casos de esplenitis por blastomicosis en perros con solo lesiones en bazo (Cuadro 1). Estos casos representan el $0.10 \%$ del total de casos de caninos analizados en los dos laboratorios de patología $(n=5896)$, de los cuales la distribución por año diagnosticada con blastomicosis esplénica fue $2015(\mathrm{n}=3), 2016(\mathrm{n}=2)$ y $2017(\mathrm{n}=1)$

Clínicamente, la mayoría de los pacientes presentaron vómito, decaimiento, inapetencia y pérdida de peso marcada. Se evidenció esplenomegalia o nodulaciones que podrían corresponder a áreas de necrosis esplénica por reacción inflamatoria de tipo piogranulomatoso en ubicación subcapsular.

En la evaluación histopatológica se encontraron lesiones y estructuras compatibles con blastomicosis (Figura 2). Además, se encontró hiperplasia nodular linfoide, así como algunos casos con marcados cambios de depleción en centros germinales.

Los casos analizados son compatibles con reacción inflamatoria crónica, evidenciable por la presencia de células gigantes y fibroplasia (Figura 2 A, D, E). Tam-
Cuadro 1. Casos de esplenitis micótica canina encontrados en los laboratorios de patología de Corpavet $(\mathrm{C}$ y la Universidad de La Salle (Colombia, 2013-2017)

\begin{tabular}{llc}
\hline Raza & Sexo & $\begin{array}{c}\text { Edad } \\
\text { (años) }\end{array}$ \\
\hline Bobtail & Hembra & 11 \\
Pitbull & Hembra & NR \\
Bulldog & NR & 4 \\
Pastor Alemán & Hembra & 2 \\
Yorkshire & Macho & 9 \\
Terrier & Macho & 13 \\
NR $^{1}$ & \\
\hline
\end{tabular}

${ }^{1}$ No registrado

bién pudo observarse la presencia de polimorfonucleares que caracterizan el proceso como piogranulomatoso (Figura $2 \mathrm{D}$ ). así como estructuras micóticas compatibles con Blastomyces (Figuras $2 \mathrm{~A}-\mathrm{F}$ ). La literatura reporta que la enfermedad granulomatosa es una de las causas de esplenomegalia firme, y los procesos neoplásicos infiltrativos son uno de los principales diagnósticos diferenciales de este tipo de 

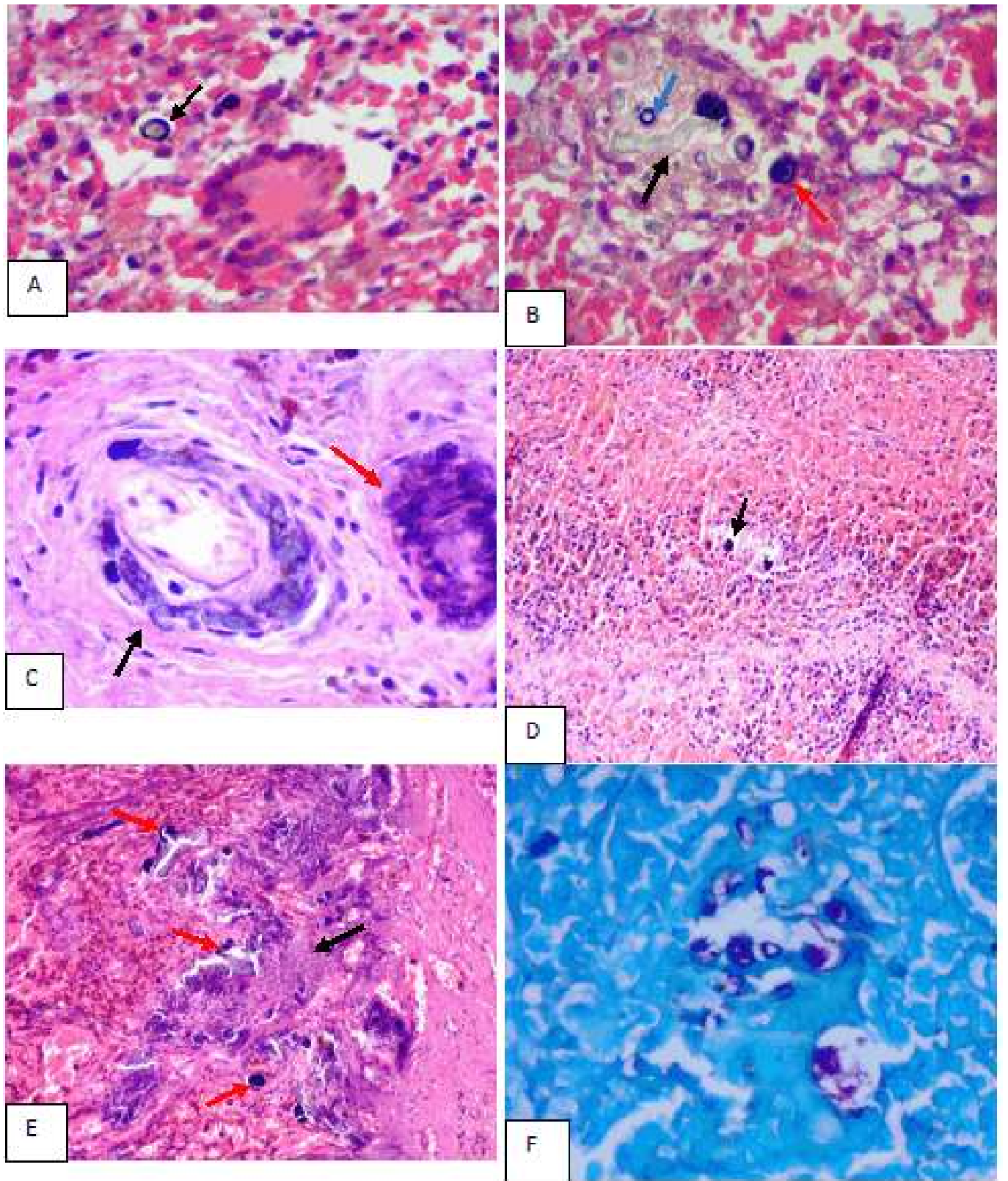

Figura 2. Cortes histológicos de bazo canino con esplenitis. A: Presencia de una célula gigante, con esporas de Blastomyces aledaña (flecha). Nótese la marcada basofilia, la pared gruesa bilaminal y el espacio entre la espora y el tejido circundante efecto de la pared refráctil. H-E. 10X. B: Estructuras micóticas compatibles con hifas hialinas (flecha negra), esporas de Blastomyces completamente formadas (flecha roja) y esporas sin formación finalizada compatibles con microformas (flecha azul). H-E. 10X. C: Infiltración perivascular de formas micóticas. Nótese que la pared vascular se encuentra intacta. Vénula (flecha negra), arteriola (flecha roja). H-E. 10X. D: Presencia de estructuras micóticas compatibles con esporas de Blastomyces (flecha negra). Se observa reacción inflamatoria asociada, caracterizada por la presencia de algunos polimorfonucleares neutrófilos y fibroplasia leve. H-E. 4X. E: Presencia de estructuras micóticas compatibles con esporas y microformas de Blastomyces (flechas rojas). Se observa reacción inflamatoria asociada, caracterizada por marcada fibroplasia (flecha negra). H-E. 4X. F: Presencia de estructuras micóticas compatibles con esporas de Blastomyces. Tinción de Grocott. 10X 
esplenomegalia (McGavin y Zachary, 2007; Couto y Nelson, 2014).

En la mayoría de los casos, los hallazgos clínicos, la exploración ecográfica y los análisis hematológicos y bioquímicos no revelaron cambios significativos que pudieran relacionarse con cuadros micóticos, posiblemente asociado a la localización en el órgano (bazo), un bajo grado de severidad y extensión y la no presentación de evidencia clínica o histopatológica de lesiones en otros órganos. Asimismo, llama la atención que estos casos eran clínicamente compatibles con sospecha de procesos neoplásicos o estados hemoparasitarios agudos, por lo que se realizaron las esplenectomías a los pacientes con el fin de extraer lo que se sospechaba era una neoplasia e identificar su origen.

Teniendo en cuenta la baja casuística encontrada $(0.101 \%, 6 / 5896$ entre 2013 y 2017) en los dos laboratorios de patología, es común que los casos de blastomicosis localizados únicamente en el bazo pasen desapercibidos y no sean tenidos en cuenta por el clínico como posible diagnóstico diferencial para procesos de esplenomegalia; sin embargo, este reporte documenta casos poco frecuentes relacionados con la presentación de esplenitis micóticas y otorga información sobre herramientas diagnósticas para el manejo de la enfermedad.

\section{Literatura Citada}

1. Acha P, Szyfres B. 2001. Zoonosis y enfermedades transmisibles al hombre y a los animales. Publicación científica y técnica No. 580. Washington, USA: OPS. 420 p.

2. [AEMV] Association of Exotic Mammal Veterinarians. 2014. Pulmonary blastomycosis in domestic ferret (Mustela putorius furio). J Exotic Pet
Med 23: 158-164. doi:/10.1053/ j.jepm.2014.02.010

3. Brömel C, Sykes J. 2005. Epidemiology, diagnosis and treatment of blastomycosis in dogs and cats. Clin Tech Small Anim Pract 20: 233-239. doi: 10.1053/ j.ctsap.2005.07.004

4. Castellani A. 1928. Notes of blastomycosis, its aetiology and clinical varieties. Proc R Soc Med 21: 447-462.

5. Couto G, Nelson R. 2014. Medicina interna de pequeos animais. $5^{\text {a }}$ ed. Sao Paulo: Elsevier. $1474 \mathrm{p}$.

6. Ettinger S, Feldman E. 2010. Textbook of veterinary internal medicine expert consult. $7^{\mathrm{a}}$ ed. Los Angeles, USA: Saunders Elsevier. 2208 p.

7. Guarner J, Brandt M. 2011. Histopathologic diagnosis of fungal infections in the 21 st century. Clin Microbiol Rev 24: 247-280. doi: 10.1128/ CMR.00053-10

8. Latimer K, Mahaffei E, Prassee K. 2005. Patología clínica veterinaria. $4^{\mathrm{a}} \mathrm{ed}$. Barcelona: Multiediciones Veterinarias. $492 \mathrm{p}$.

9. McGavin D, Zachary J. 2007. Pathologic basis of veterinary disease. $4^{\mathrm{a}}$ ed. St. Louis, USA: Mosby-Elsevier. $1476 \mathrm{p}$.

10. Patel J, Gatusso P, Vijaya B. 2010. Diagnosis of blastomycosis in surgical pathology and cytopathology: correlation with microbiologic culture. Am J Surg Pathol 34: 256-261. doi: 10.1097/ PAS.0b013e3181ca48a5.

11. Quinn P, Markey K, Leonard F, Fitzpatrick S, Fanning S. 2003. Concise review of veterinary microbiology. $2^{\mathrm{a}}$ ed. Great Britain: Blackwell Publishing. 208 p.

12. Ramanan P, Wengenack $N$, Theel ES. 2017. Laboratory diagnostics for fungal disease: a review of current and future diagnostic assays. Clin Chest Med 38: 535-554. doi: 10.1016/j.ccm.2017.04.013 clinic test. Med 38: 535-554 
13. Raymond J, White R, Kilbane T, Janovitz J. 1997. Pulmonary blastomycosis in an Indian fruit bat (Pteropus giganteus). J Vet Diagn Invest 9: 8587. doi: 10.1177/104063879700900117

14. Sánchez L, Cabanilla J. 2010. Infec ciones micóticas sistémicas o profundas:blastomicosis norteamericana. Dermatol Peru 20: 250-256.

15. Stenske K. 2008. Update of diagnostic options for canine blastomycosis. Adv Small Anim Med Surg 21: 1-3. 